\title{
Design of manufacturable 3D extremal elastic microstructure
}

\section{Andreassen, Erik; Lazarov, Boyan Stefanov; Sigmund, Ole}

\section{Published in:}

Mechanics of Materials

Link to article, DOI:

10.1016/j.mechmat.2013.09.018

Publication date:

2014

Document Version

Peer reviewed version

Link back to DTU Orbit

Citation (APA):

Andreassen, E., Lazarov, B. S., \& Sigmund, O. (2014). Design of manufacturable 3D extremal elastic microstructure. Mechanics of Materials, 69, 1-10. https://doi.org/10.1016/j.mechmat.2013.09.018

\section{General rights}

Copyright and moral rights for the publications made accessible in the public portal are retained by the authors and/or other copyright owners and it is a condition of accessing publications that users recognise and abide by the legal requirements associated with these rights.

- Users may download and print one copy of any publication from the public portal for the purpose of private study or research.

- You may not further distribute the material or use it for any profit-making activity or commercial gain

- You may freely distribute the URL identifying the publication in the public portal

If you believe that this document breaches copyright please contact us providing details, and we will remove access to the work immediately and investigate your claim 


\title{
Design of manufacturable 3D extremal elastic microstructure
}

\author{
Erik Andreassen*, Boyan S. Lazarov, Ole Sigmund \\ Department of Mechanical Engineering, Technical University of Denmark, Nils Koppels \\ Allé, Building 404, Denmark
}

\begin{abstract}
We present a method to design manufacturable extremal elastic materials. Extremal materials can possess interesting properties such as a negative Poisson's ratio. The effective properties of the obtained microstructures are shown to be close to the theoretical limit given by mathematical bounds, and the deviations are due to the imposed manufacturing constraints. The designs are generated using topology optimization. Due to high resolution and the imposed robustness requirement they are manufacturable without any need for post-processing. This has been validated by the manufacturing of an isotropic material with a Poisson's ratio of $\nu=-0.5$ and a bulk modulus of $0.2 \%$ times the solid base material's bulk modulus.
\end{abstract}

Keywords: topology optimization, auxetic material, microstructure, additive manufacturing, inverse homogenization

\section{Introduction}

The focus of this paper is on the design of elastic three-dimensional materials with periodic microstructures manufacturable by additive manufacturing techniques like selective laser sintering (SLS) (see Kalpakjian et al., 2010, for a description of the technique). The microstructures are built from a single material with voids, and effective material properties are found by numerical homogenization (Guedes and Kikuchi, 1990). The designs are obtained using topology optimization without any post-processing by requiring their performance to be insensitive with respect to uniform variations of the

\footnotetext{
*E-mail: erand@mek.dtu.dk
} 
geometry like erosion or dilation (over- or under-etching). The requirement for robustness leads to the definition of a minimum length scale in the topology (Wang et al., 2011), and therefore any imperfections with dimensions smaller than the defined length scale, which might appear due to uncertainties in the production process, do not significantly affect the effective material properties on macroscale.

The topology optimization method is an iterative design process, which optimizes a material distribution in a given design domain with respect to a specified objective function and a set of constraints. It is utilized in a broad range of problems varying from pure mechanical designs (e.g. aerospace structures (Krog et al., 2004)) to designs in electromagnetics and photonics (e.g. Jensen and Sigmund, 2011). A popular introduction to the method can be found in (Sigmund, 2000b), while a thorough description is given in (Bendsøe and Sigmund, 2003). Already in (Sigmund, 1994) it is shown that topology optimization can be used to design materials with a prescribed elasticity tensor by inverse homogenization. Several papers followed on the design of elastic materials (e.g. Swan and Kosaka, 1997; Theocaris and Stavroulakis, 1998) as well as (Sigmund, 2000a), where a new class of extremal composites were presented. Gibiansky and Sigmund (2000) demonstrate the method on the design of three-phase elastic materials, and Sigmund and Torquato (1999) extend this to obtain designs with prescribed thermal and electrothermal properties. Designs optimized for multiple properties, such as fluid permeability and stiffness are shown by Guest and Prévost (2006).

The application of topology optimization to design structural materials is an active field of research. Recent examples are given by Coelho et al. (2011), who demonstrate how a multi-scale formulation can be used to design a trabecular bone section, and Diaz and Sigmund (2010), who apply topology optimization to design a so-called metamaterial with negative permeability (electromagnetic). A thorough review of microstructure design by topology optimization is given by Cadman et al. (2012).

Here, the procedure is exemplified by considering the design of manufacturable extremal elastic materials and negative Poisson's ratio materials (auxetic materials). Several examples of two-phase two-dimensional structures with negative Poisson's ratio exist in the literature. Milton (1992) is the first who found a family of two-dimensional materials with Poisson's ratio arbitrarily close to -1 within the framework of laminated elastic materials. Later, Sigmund (2000a) shows that topology optimization can be applied to the design of both two- and three-dimensional isotropic materials with nega- 
tive Poisson's ratio. However, the obtained designs are not always manufacturable due to the thin hinge connections in the microstructure. Topology optimization can also be applied to decrease Poisson's ratio between two of the principal directions (Sigmund et al., 1998; Schwerdtfeger et al., 2011) for anisotropic materials.

Theoretically, all achievable effective elastic material tensors can be generated using so-called pentamode material microstructure (Milton and Cherkaev, 1995), which possess parts connected by infinitely thin hinges. These theoretical designs can hardly be produced, and their manufacturability for a modified version with a finite extent is explored in a recent paper by Kadic et al. (2012). The topology optimization procedure presented here ensures manufacturability of the resulting microstructure. Furthermore, we demonstrate that the optimized robust designs can reach the mathematical bounds for three-dimensional isotropic materials in certain cases.

In the next section the mathematical formulation of the optimization problem for the minimization of Poisson's ratio is stated. A slightly modified formulation that can be used to optimize for other extreme material properties is explained as well. In Section 3 the method used to achieve manufacturable designs is described and illustrated. The optimized structures are presented and discussed in Section 4 together with measurements on a manufactured version of the isotropic negative Poisson's ratio material.

\section{Optimization problem formulation}

The objective and the constraints in the design process are calculated by extracting material properties from the macroscale elasticity tensor. The material is constructed by repeating a unit cell in all spatial directions. The design is periodic and the effective elasticity tensor is obtained using numerical homogenization (e.g. Guedes and Kikuchi, 1990). A compact description of the process is included in Appendix A.

The unit cell is discretized using first-order finite elements. A design variable is associated with each element, which takes values between zero (void) and one (element filled with material). A modified SIMP scheme (Bendsøe and Sigmund, 2003), with a constant penalization factor of 3, is used to interpolate between void and solid in each finite element. The continuous design variables are updated by a standard topology optimization approach. A full description of the method is outside the scope of the current paper and here only the steps important to the design of material microstructures 
are discussed in details. A compact educational implementation of a typical topology optimization procedure can be found in (Andreassen et al., 2011).

\subsection{Minimizing Poisson's ratio}

The optimization problem for minimizing the effective Poisson's ratio $\nu^{*}$ can be stated as:

$$
\begin{aligned}
& \min : \quad \nu^{*} \quad \text { Poisson's ratio } \\
& \text { s. t.: } \quad \mathbf{K} \chi^{i}=\mathbf{f}^{i}, \quad i=1, . ., 6 \quad \text { Homogenization equations } \\
& K^{*} \geq a K, \quad \text { Bulk constraint } \\
& \frac{\sum_{i, j}\left(C_{i j}^{i s o}-C_{i j}^{*}\right)^{2}}{\left(C_{11}^{i s o}\right)^{2}} \leq \varepsilon, \quad i, j=1, \ldots, 6 \quad \text { Isotropy constraint } \\
& \frac{1}{|\Upsilon|} \sum_{e}\left(v_{e} \rho_{e}\right) \leq V, \quad e=1, \ldots, N \quad \text { Volume constraint } \\
& 0 \leq \rho_{e} \leq 1, \quad e=1, \ldots, N \quad \text { Element densities }
\end{aligned}
$$

where $\boldsymbol{\rho}$ is a vector of element densities, and $\nu^{*}$ is the effective Poisson's ratio extracted from the homogenized elasticity tensor. $\mathbf{K}$ is the finite element stiffness matrix for the discretized unit cell and $\chi^{i}$ are the displacement vectors corresponding to six (three in 2D) different unit load cases $\mathbf{f}^{i}$ (for more details see Appendix A). $K^{*}$ is the homogenized bulk modulus, $K$ is the bulk modulus of the solid phase, $a$ is the required minimum ratio

between bulk modulus of solid material and microstructure. $C_{i j}^{*}$ and $C_{i j}^{i s o}$ are entries in the homogenized constitutive matrix and a corresponding isotropic constitutive matrix, respectively. $\varepsilon$ is a small number (e.g. $\left.10^{-5}\right),|\Upsilon|$ is the volume of the considered unit cell, $v_{e}$ is the volume of each finite element in the unit cell, $\rho_{e}$ is the corresponding density, and $V$ is the ratio between the allowed solid material volume and the total unit cell volume.

The bulk modulus constraint ensures a minimum stiffness of the material. An alternative formulation would be to impose a limit on the effective Young's modulus. The isotropy constraint ensures that the material proper- 
ties are isotropic in all spatial directions. $\mathbf{C}^{i s o}$ is defined as:

$$
\begin{aligned}
& C_{i i}^{i s o}=\left(C_{11}^{*}+C_{22}^{*}+C_{33}^{*}\right) / 3, \quad i=1,2,3 \\
& C_{i j}^{i s o}=\left(C_{12}^{*}+C_{13}^{*}+C_{23}^{*}\right) / 3, \quad i, j=1,2,3, \quad i \neq j \\
& C_{i i}^{i s o}=\left(C_{11}^{i s o}-C_{12}^{i s o}\right) / 2, \quad i=4,5,6 \\
& C_{i j}^{i s o}=0 \quad \text { else }
\end{aligned}
$$

where $\mathbf{C}^{*}$ is the homogenized constitutive matrix. The isotropy constraint can be changed to a cubic symmetric constraint by substituting Equation (4) with

$$
C_{i i}^{i s o}=\left(C_{44}^{*}+C_{55}^{*}+C_{66}^{*}\right) / 3, \quad i=4,5,6
$$

The nonlinear optimization problem defined by Equation (1) is solved using the gradient-based Method of Moving Asymptotes (MMA) (Svanberg, 1987) with analytical sensitivity computations. The topology optimization problem is regularized by a projection scheme combined with the so-called robust optimization formulation (Wang et al., 2011; Lazarov et al., 2011) which ensures black and white $(0 / 1)$ designs with a minimum length scale. When using a projection approach, it is advisable to gradually increase the projection parameter $\beta$ (going from a linear function to a step function), and we use a continuation scheme similar to the one in Wang et al. (2011); however, the continuation scheme is only applied after the design has converged with an initial $\beta=1$. Filters and projection methods are discussed in depth by Sigmund (2007) and more details on the robust optimization scheme are given in Section 3 .

\subsection{Analytic bounds for isotropic materials}

By substituting the objective function in Equation (1) with a maximization of the effective shear modulus $G^{*}$ and varying $a$, or alternatively maximizing $K^{*}$ and having a constraint on $G^{*}$, material structures close to the Hashin-Shtrikman bounds can be found. For an isotropic material with microstructure consisting of one material with voids the upper HashinShtrikman bounds (Hashin and Shtrikman, 1963) on the effective bulk $K^{*}$ and effective shear modulus $G^{*}$ are given as: 


$$
\begin{aligned}
K^{*} & \leq \frac{4 G K V}{4 G+3 K(1-V)}, \\
G^{*} & \leq G+\frac{1-V}{\frac{6}{5 G} \frac{(K+2 G) V}{(3 K+4 G)}-\frac{1}{G}},
\end{aligned}
$$

where $V$ is the volume fraction of base material, $K$ the bulk modulus, and $G$ the shear modulus of the base material. The corresponding lower bounds are zero. It should be pointed out that the upper limit on $K^{*}$ also holds for cubic symmetric materials. For two-dimensional materials the improved Cherkaev-Gibiansky bounds (Cherkaev and Gibiansky, 1993) can be used as well. Berryman and Milton (1988) have presented improved bounds correlating $K^{*}$ and $G^{*}$ for two-phase isotropic three-dimensional materials, however, for the case considered here, where one phase is void, those bounds coincide with the Hashin-Shtrikman bounds.

\section{Manufacturable design}

The designs obtained by standard topology optimization without projection and the requirement for robustness possess gray scale, i.e., design values between zero and one. Such solutions of the optimization problem require post-processing before manufacturing. For two-dimensional structures it is relatively easy to manually correct the design and verify it. However, for three-dimensional structures, with complex designs, the post-processing and the verification process can be prohibitive. Therefore, to be of real practical use when designing microstructures, the topology optimization process must result in designs ready for manufacturing. A standard smoothing or thresholding might be necessary to generate an STL-file for the SLS process, but preferably no manual decision-making, as this can easily violate the constraints imposed on the design and decrease performance.

\subsection{Isotropic minimum Poisson's ratio design}

The first examples where Poisson's ratios are minimized are two-dimensional, as it is relatively easy to illustrate the effect of lack of robustness here. A base material with $\nu=0.3$ is considered in the optimization process. Designs with density and sensitivity filters obtained without any manufacturing constraints are shown in Figure 1. The design in Figure 1a is a result of an 


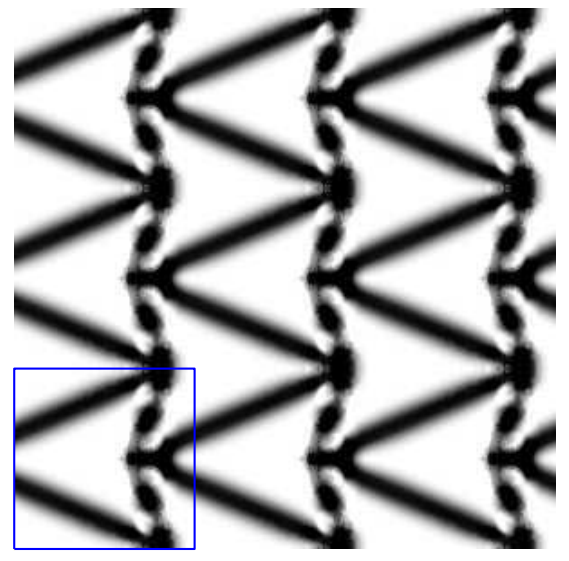

(a)

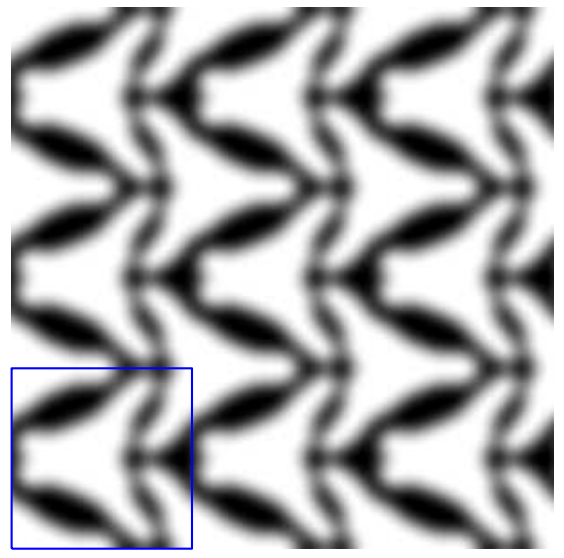

(b)

Figure 1: Minimum Poisson's ratio design obtained with standard filtering. In both cases a linearly decaying filter with a radius of $1 / 10$ of the unit cell side length was used. Other parameters are: $a=0.2 \%, \epsilon=10^{-5}, N=10000$, and $V=35 \%$. (a) Sensitivity filter started from a random distribution of densities. (b) Density filter started from the design in (a). The box in the corner encloses one unit cell.

optimization with a random initial distribution of densities. To make the visual comparison of the microstructures straightforward, the optimizations for the other $2 \mathrm{D}$ designs presented in this initial example (Figure 1b and 2b) have been run with the design from Figure $1 \mathrm{a}$ as an initial guess. Starting with a random guess results in similar but shifted or flipped designs, obscuring the possibility of direct comparisons. All the three-dimensional designs presented in Section 4 are results of optimizations with random initial density distributions. The constitutive matrices for the microstructures in Figure 1 are:

$\mathbf{C}_{a}^{*}=10^{-2} E \cdot\left(\begin{array}{rrr}1.50 & -1.21 & 0.00 \\ -1.21 & 1.50 & 0.00 \\ 0.00 & 0.00 & 1.35\end{array}\right), \mathbf{C}_{b}^{*}=10^{-2} E \cdot\left(\begin{array}{rrr}0.77 & -0.49 & 0.00 \\ -0.49 & 0.77 & 0.00 \\ 0.00 & 0.00 & 0.63\end{array}\right)$

which imply isotropic materials with Poisson's ratios of

$$
\nu_{a}^{*}=-0.81, \nu_{b}^{*}=-0.64
$$


However, the gray transition areas, imposed by the filters, correspond to porous regions. It is not possible to manufacture these designs without postprocessing, and it is not possible to say a priori how the post-processing will change the constitutive matrix. Due to the nature of the filters these areas are more pronounced for the density filter despite that the same filter radius was used for both cases.

It shall be pointed out that the designs in Figure 1 have been generated with a constant filter size, as opposed to the designs in e.g. (Sigmund, 1994), where the filter radius was gradually decreased during the optimization process. With a gradually decreasing filter radius both designs would become black and white, but at the risk of loosing the length scale and potentially getting one-node hinges (infinitely thin structures). The same holds for the case where a simple projection is used on top of the filter (Sigmund, 2007; Wang et al. 2011), as discussed in the next section.

\subsection{Black and white design}

In Figure $2 \mathrm{a}$ the microstructure resulting from an optimization where a projection is used on top of the density filter is shown. The resulting design become black and white, and has the constitutive matrix

$$
\mathbf{C}^{*}=10^{-2} E \cdot\left(\begin{array}{rrr}
2.81 & -2.53 & 0.00 \\
-2.53 & 2.81 & 0.00 \\
0.00 & 0.00 & 2.67
\end{array}\right)
$$

implying a Poisson's ratio of -0.90. The lower bound on the bulk modulus prevents one node hinges from forming, and, thus, removing a tiny strip of material from the structure uniformly (eroding it), would decrease the Poisson's ratio further. This is illustrated in Figure 3a, however, at some point the material would become disconnected, because the hinges are completely eroded away and connectivity lost. As one can deduce from Figure 3a, this happens around a volume fraction of 0.33 . When the material becomes disconnected, it does not make sense to talk about a Poisson's ratio, but since we model void as an extremely soft material, it can still be computed. Increasing the material in the structure uniformly (dilating it) will make the hinges less pronounced and this is reflected in a higher Poisson's ratio in Figure 3a. Furthermore, the only point on the graph in Figure 3a that satisfies the isotropy constraint is the one we optimized for $(V=35 \%)$. How 
to achieve a microstructure that is robust (for both the objective and the constraints), w.r.t. to removing or adding material, is illustrated in the next section.

\subsection{Robust design}

Manufacturable designs can be obtained using the approach suggested in (Sigmund, 2009; Wang et al., 2011), which is referred to as deterministic robust formulation. The optimized designs in this formulation are required to perform equally well if they are eroded or dilated. The objective function is substituted with

$$
\min _{\boldsymbol{\rho}}: \max \left(\nu^{*}, \nu_{d}^{*}, \nu_{e}^{*}\right),
$$

where $\nu_{d}^{*}$ and $\nu_{e}^{*}$ are the effective Poisson's ratios of the dilated and eroded structures, and $\nu^{*}$ is the effective Poisson's ratio of the blueprint (intermediate) design supplied to the manufacturer. The three designs are required to be isotropic. The filter radius of 0.10 is the same as before, which combined with the applied projection thresholds ensures a minimum length scale of 0.09 (see Wang et al., 2011). The bulk modulus constraint is applied only on the eroded case, since it is the weakest of the three structures. The deterministic robust formulation results in a design shown in Figure 2b, with an effective constitutive tensor

$$
\mathbf{C}^{*}=10^{-2} E \cdot\left(\begin{array}{rrr}
2.40 & -1.85 & 0.00 \\
-1.85 & 2.40 & 0.00 \\
0.00 & 0.00 & 2.12
\end{array}\right)
$$

which corresponds to a Poisson's ratio of -0.77 .

In Figure $3 \mathrm{~b}$ the dependence of the objective with respect to uniform dilation or erosion is illustrated. Eroding the design further than the selected erosion threshold improves the effective Poisson's ratio. This effect is due to the bulk modulus constraint. Without it, hinges appear in the eroded design and the performance deteriorates immediately.

For the design with the objective shown in Figure 3b it seems that only the dilated structure is active in the $\min / \max$ objective function, hence, one might be tempted to select only the dilated case in the optimization process. However, we have observed that such a reduced formulation can lead to poor performance since during the first few hundred iterations all three designs play a role in the design update. Furthermore, the isotropy

constraint is active on all three designs. Since manufacturing tolerances 


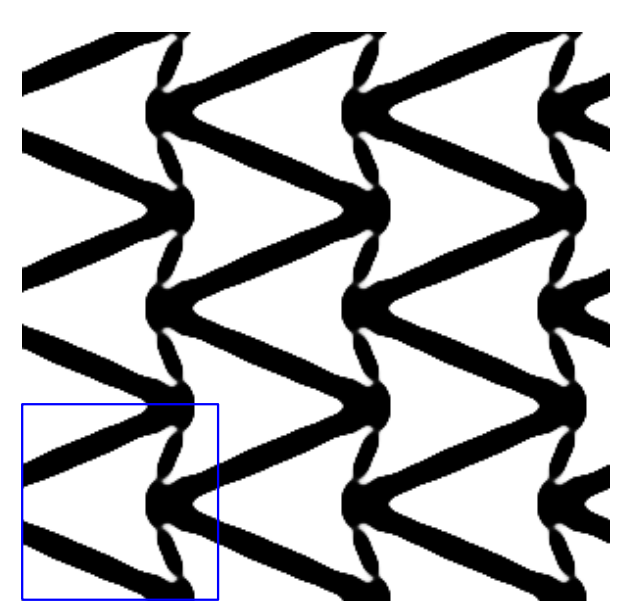

(a)

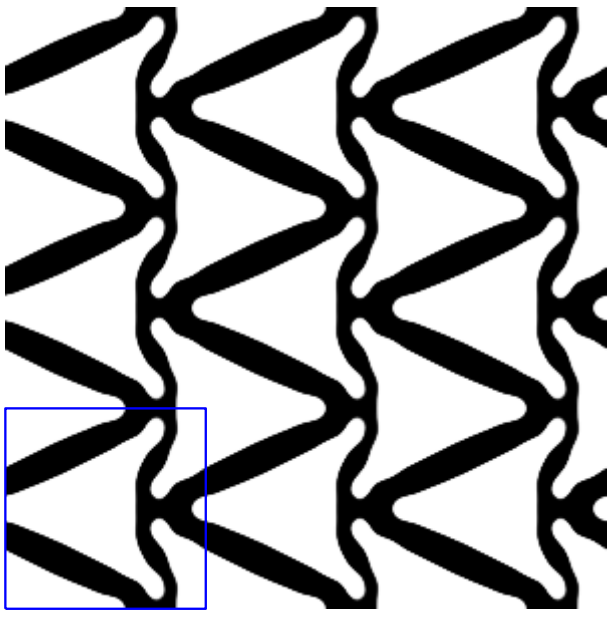

(b)

Figure 2: Negative Poisson's ratio structures obtained with (a) a projection, and (b) robust formulation. Parameters are the same as for the designs in Figure 1, except for the volume constraint in the robust formulation, which is applied on the dilated design and is set 15 percent higher. The design in Figure $1 \mathrm{a}$ is used as the initial guess.

are taken into account, Poisson's ratios very close to -1 are not achievable (pure hinges are prohibited, cf. (Kadic et al., 2012)), however the blueprint design is manufacturable without any amendments. Materials with Poisson's ratios of -1 can be created with mechanism-like structures built from trusses connected through hinges that do not resist bending; imperfect hinges will limit the achievable Poisson's ratio.

As discussed by Wang et al. (2011) a minimum length scale is assured as long as the topologies of the three designs are the same. Obtaining the same topologies can be a challenge and difference in the topologies can severely affect the performance. Including more projections in the optimization objective can give smoother transition between the designs. This case can be naturally handled by modeling the projection threshold as a random variable. Such a formulation is presented by Lazarov et al. (2011) and is the one we implemented and utilized for the three-dimensional designs discussed in the next section. However, comparing designs from runs with more than three design realizations and runs with only three design realizations, little difference was found. Thus, for all the three-dimensional designs presented in 


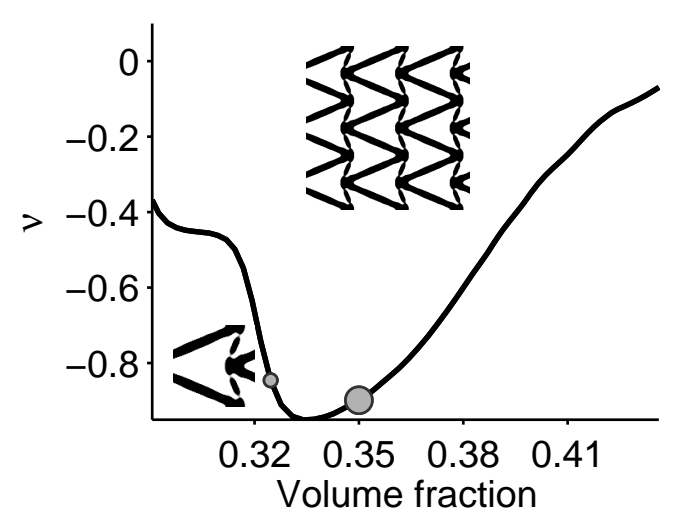

(a)

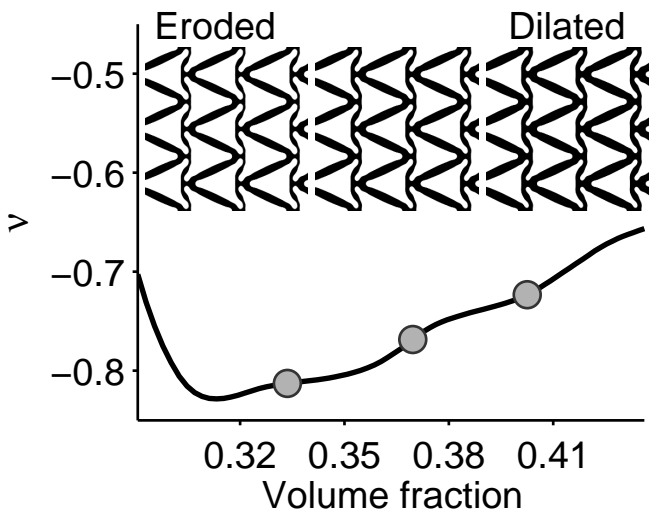

(b)

Figure 3: Graphs illustrating variation of the effective Poisson's ratio with respect to erosion/dilation of the designs in (a) Figure 2a-projected design, and (b) Figure 2b-robust design.

the next section, three designs (eroded, blueprint, and dilated) were included in the optimization.

\section{Three-dimensional design}

The two-dimensional designs presented above illustrate how manufacturable material designs can be achieved. The approach is directly applicable to three dimensions, with the main issue being the computational cost. Therefore, the optimization results presented below have been obtained using a parallel implementation of the optimization process (Aage and Lazarov, 2013).

Our main example is a three-dimensional isotropic negative Poisson's ratio material, which has been manufactured in polyamid using SLS. Several three-dimensional designs close to the theoretical limits mentioned in Section 2.2 are presented as well. Finally, we briefly discuss the computational cost.

\subsection{Isotropic minimum Poisson's ratio}

The structure is manufactured using polyamid with Poisson's ratio 0.4, which is the base material in the optimization process. The $3 \mathrm{D}$ material microstructure resulting from the optimization process is shown in Figure 4. The effective constitutive tensor is: 


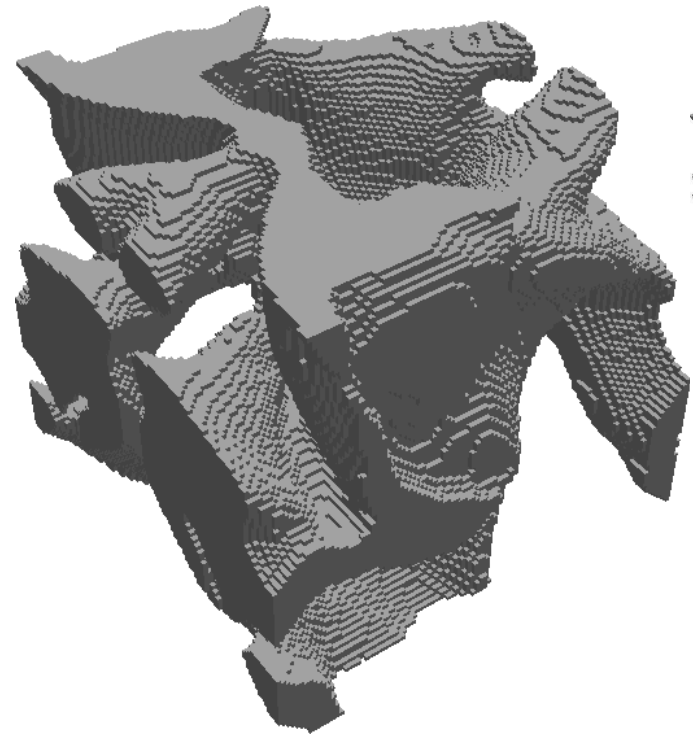

(a)

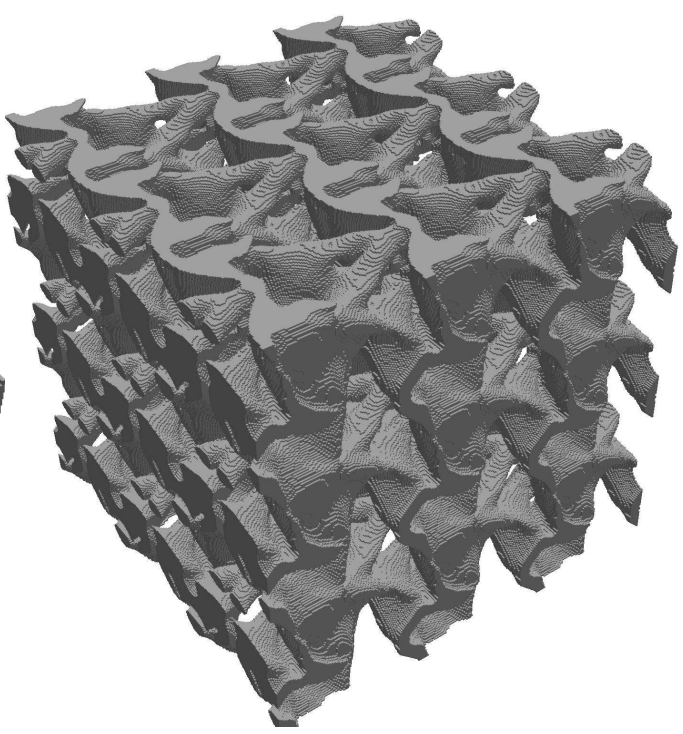

(b)

Figure 4: 3D isotropic microstructure with Poisson's ratio $-0.51 . a=0.125 \%$ is used for the stiffness constraint on the eroded design, while the isotropy constraint parameter $\epsilon$ is set to $10^{-5}$ for all three designs. The solid volume fraction of the dilated design is constrained to $40 \%$. The initial guess is a random distribution of densities. (a) One unit cell. (b) $3 \times 3 \times 3$ unit cells.

$$
\mathbf{C}^{*}=10^{-2} E_{\text {polyamid }}\left(\begin{array}{rrrrrr}
3.42 & -1.15 & -1.15 & 0.00 & 0.00 & 0.00 \\
-1.15 & 3.42 & -1.15 & 0.00 & 0.00 & 0.00 \\
-1.15 & -1.15 & 3.42 & 0.00 & 0.00 & 0.00 \\
0.00 & 0.00 & 0.00 & 2.28 & 0.00 & 0.00 \\
0.00 & 0.00 & 0.00 & 0.00 & 2.28 & 0.00 \\
0.00 & 0.00 & 0.00 & 0.00 & 0.00 & 2.28
\end{array}\right) \text {, }
$$

which implies an isotropic material with a Poisson's ratio of

$$
\nu=-0.51
$$

The only physically realized three-dimensional isotropic material presented in the literature with a comparable Poisson's ratio is Lakes' reentrant foam (see e.g. Lakes, 1987). However, compared to the presented 3D microstructure, the foam materials have a much lower bulk modulus. For the polyester foam in (Lakes, 1987) $K^{*} / K \approx 10^{-6}$ (where $K$ is taken for pure polyester), while 
$K^{*} / K \approx 2 \cdot 10^{-3}$ for the structure in Figure 4. It should be mentioned that Lakes' design was a conceptual development - proving that it is indeed possible to produce negative Poisson's ratio material - based on insight, and not the result of an analytical approach.

\subsection{Experimental test}

To validate the manufacturability of the three-dimensional design the isotropic negative Poisson's ratio material is produced in polyamid using an SLS machine and Poisson's ratio is measured using a simple compressive test. A photo of the manufactured structure can be seen in Figure 7. It consists of $8 \times 4 \times 4$ unit cells, with side lengths of $2 \mathrm{~cm}$. The aspect ratio of 2 is chosen to reduce the effect of the friction from the end surfaces when the sample is compressed. Requiring an aspect ratio of 2, a specimen with $8 \times 4 \times 4$ cells was the biggest that could be manufactured in the SLS machine.

The experimental setup is shown in Figure 5. The loading is provided by a hydraulic press while extensometers with a sensitivity of $0.01 \mathrm{~mm}$ are used to measure the deformation of the sample along the longest and perpendicular lines located at the center of the sample. In the setup the load on the sample could not be accurately controlled, so to assure full contact between all surfaces before starting the measurements, a small prestress force is applied. In order to avoid damage to the structure the load is increased six times with the aim of achieving maximal vertical strain below one percent. After each load increment the horizontal and vertical deflection values are recorded. The deflection curves are shown in Figure 6. The two plots correspond to the two short directions of the sample (the second direction is obtained by rotating the sample 90 degrees around the long axis and is perpendicular to the first).

As mentioned earlier the extensometers sensitivity is $\delta=0.01 \mathrm{~mm}$, hence this, being a quantifiable source of measurement error, has been used to generate the error curves in Figure 6. The upper curve is found by subtracting $2 \delta$ from the horizontal displacements and adding $1 \delta$ to the vertical displacements, while the lower curve is found by adding $2 \delta$ to the horizontal displacements and subtracting $1 \delta$ from the vertical displacements. Considering the last measurement point, which has the smallest relative error, the measured Poisson's ratio of the material is $-0.50 \pm 0.03$ for both sets of measurements. Furthermore, there is only one measurement point where the measured interval for Poisson's ratio does not contain -0.51 . Possible

reason for this behavior is the influence of the boundary conditions. The 


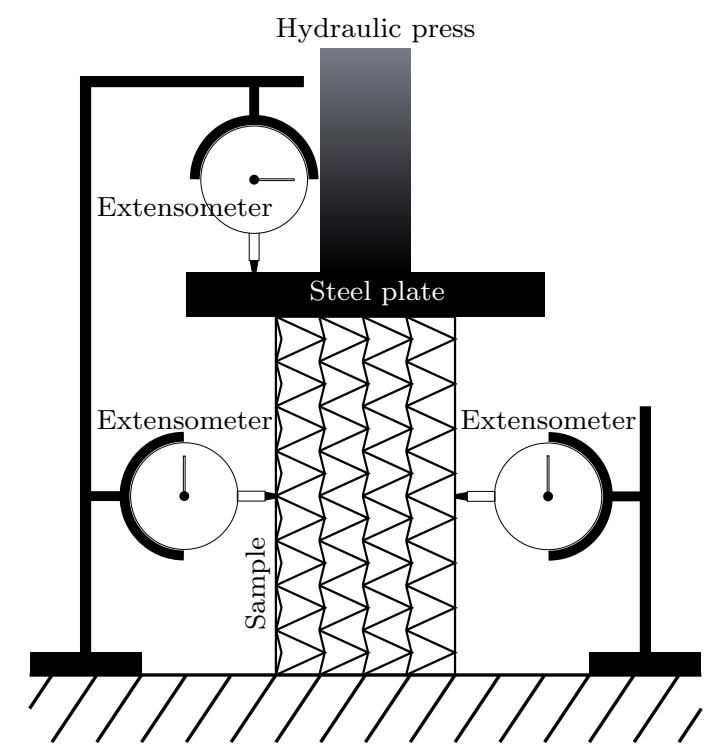

Figure 5: Experimental setup for measurements of Poisson's ratio.

homogenization theory assumes an infinite extent of the material while the sample only contains a finite number of unit cell. Other possible sources of error are the local deformations at the measurement points for the two horizontally aligned extensometer. To compensate for this we positioned them on two opposite flat areas of the structure. It should be emphasized that little influence on the measurements is observed if these extensometers were repositioned, e.g., moved one unit cell vertically or horizontally. The results presented here are from a single measurement and not an average of several measurements.

In Figure 8 close-ups of the undeformed and deformed structure are compared. It is clearly visible how the structure contracts in the horizontal direction when compressed in the vertical direction. As already stated no post-processing is performed on the structure before sending it to manufacturing and an STL-file of the unit cell is available for download from our webpage www .topopt.dtu.dk/negativePoissonsRatio/unitCell.stl.

Ideally, the experimental test should consist of repeated measurements on multiple specimens, but due to the manufacturing cost this was not feasible. However, we encourage other researchers with manufacturing capabilities to download the STL-file and conduct more advanced experiments. 


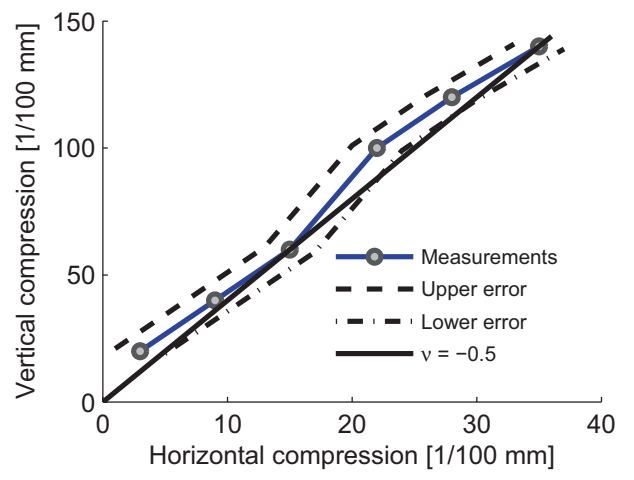

(a)

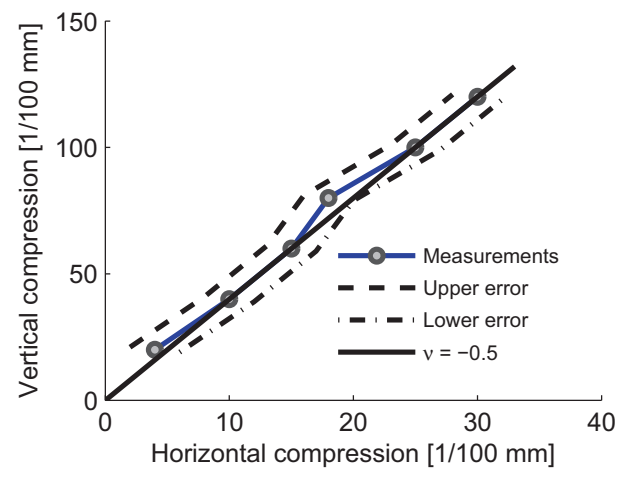

(b)

Figure 6: Deflection curves from measurements of Poisson's ratio. In (a) the sample was rotated 90 degrees around its longest axis compared to (b).

\subsection{Theoretical limits}

The negative Poisson's ratio problem is used as a case example, because it is difficult to obtain a manufacturable design. A negative Poisson's ratio material requires a large shear to bulk modulus ratio. The bulk and shear modulus of the material in Figure 4 are plotted as a point (denoted Fig. 4) together with the theoretical bounds in Figure 9. It can be seen that the design is in the lower left corner. Designs can be obtained in the upper left corner (cf. pentamode Milton and Cherkaev, 1995), however, it will be difficult to achieve manufacturabiliy for them. Several optimization runs are performed to verify this conclusion. The resulting bulk and shear moduli of these extremal materials are also shown in Figure 9. It should be pointed out that the plot shows the moduli for the middle design which is different than the mean moduli. For small erosion or dilation it will not change significantly due to the required robust performance. The hatched area in Figure 9 indicates where isotropic materials with a negative Poisson's ratio can be found.

First, consider the designs obtained with an isotropy constraint $(\epsilon=$ $10^{-5}$ ). The unit cells for the material designs corresponding to the points in the graph are shown in Figure 10. The design corresponding to point a) is just a disconnected unit cell without any stiffness. Setting constraint on the bulk modulus and maximizing the shear modulus leads to the designs shown in b), c) and d), where b) and c) have topologies similar to the negative Poisson's ratio cell. Maximizing the bulk modulus and constraining the shear modulus 


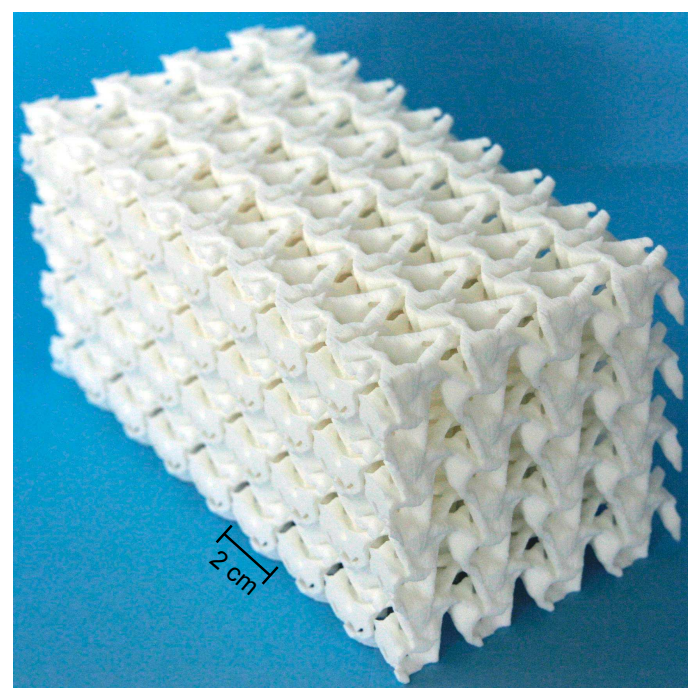

Figure 7: Manufactured minimum Poisson's ratio structure.

leads to the designs shown in h), g) and f). Maximizing the bulk modulus without any constraints results in a Vigdergauz-like cell (Vigdergauz, 1989).

Also included in Figure 9 and 10 are two designs, points i) and j), for which instead of an isotropy constraint a cubic symmetry constraint was applied in the optimization (still with $\epsilon=10^{-5}$ ). This is done to illustrate that even for structures with cubic symmetry the upper left and lower right corner are unachievable when the requirement for robustness is imposed in the optimization.

Finally, it should be mentioned that a cubic unit cell is not necessarily the best choice for design of isotropic materials. In 2D more extremal designs can sometimes be obtained with a rectangular unit cell (Sigmund, 2000a). In $3 \mathrm{D}$ it is not clear what cell shape would be most beneficial. Due to the high computational cost we have only utilized a cubic unit cell in our numerical experiments.

\subsection{Computational issues}

Design of three-dimensional structures requires extensive computational power, since the number of degrees of freedom explodes (the curse of dimensionality). A two-dimensional problem with $100^{2}$ elements will have 20000 degrees of freedom (dofs). In 3D discretizing the unit cell with $100^{3}$ elements equates to three million dofs. 


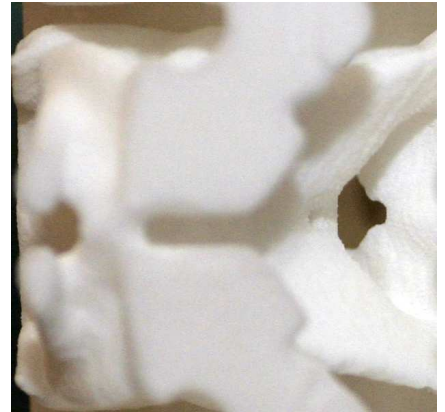

(a)

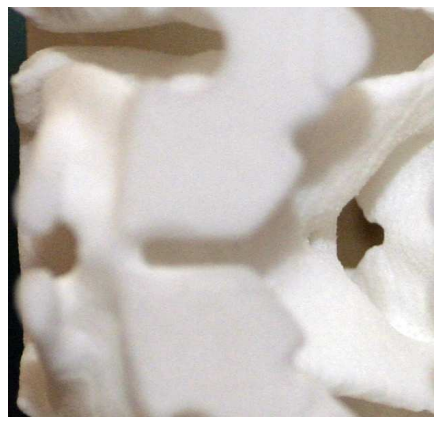

(b)

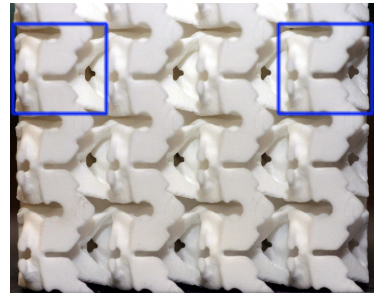

(c)

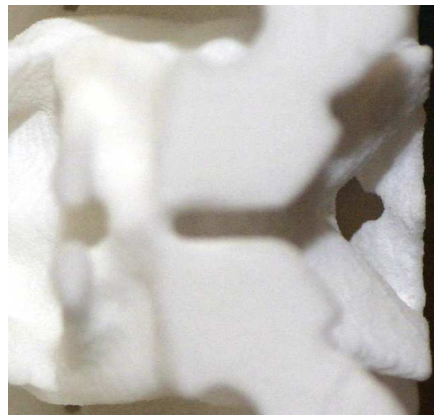

(d)

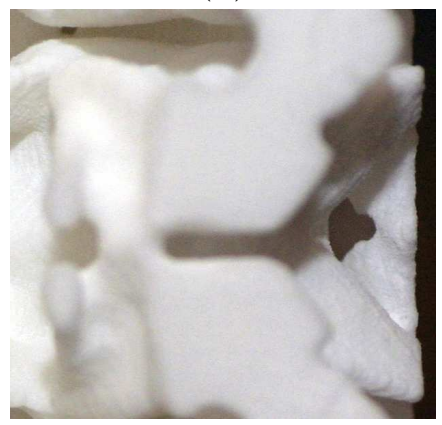

(e)

Figure 8: Visualization of the structure deformations. (a) Undeformed unit cell on left side, (b) deformed version. (d) Undeformed unit cell on right side, (e) deformed version. (c) Three bottom rows of the sample. The blue selections indicate the positions of the close ups. The negative Poisson's ratio behavior is illustrated by how both sides contract (the black edge area becomes larger).

The number of iterations in each optimization run varies, but all the optimization results in this paper took more than 1000 iterations. In each iteration three state problems are solved (eroded, blueprint, and dilated design), and an optimization run with 1000 iterations takes approximately 10 hours on 120 CPU cores.

The time per iteration is not constant, because the solutions of the state problems are obtained iteratively using a preconditioned conjugate gradient method (PCG). Its convergence depends on the effectiveness of the supplied preconditioner. The classical preconditioning techniques like incomplete factorization, diagonal and block-diagonal scaling cannot ensure a con- 


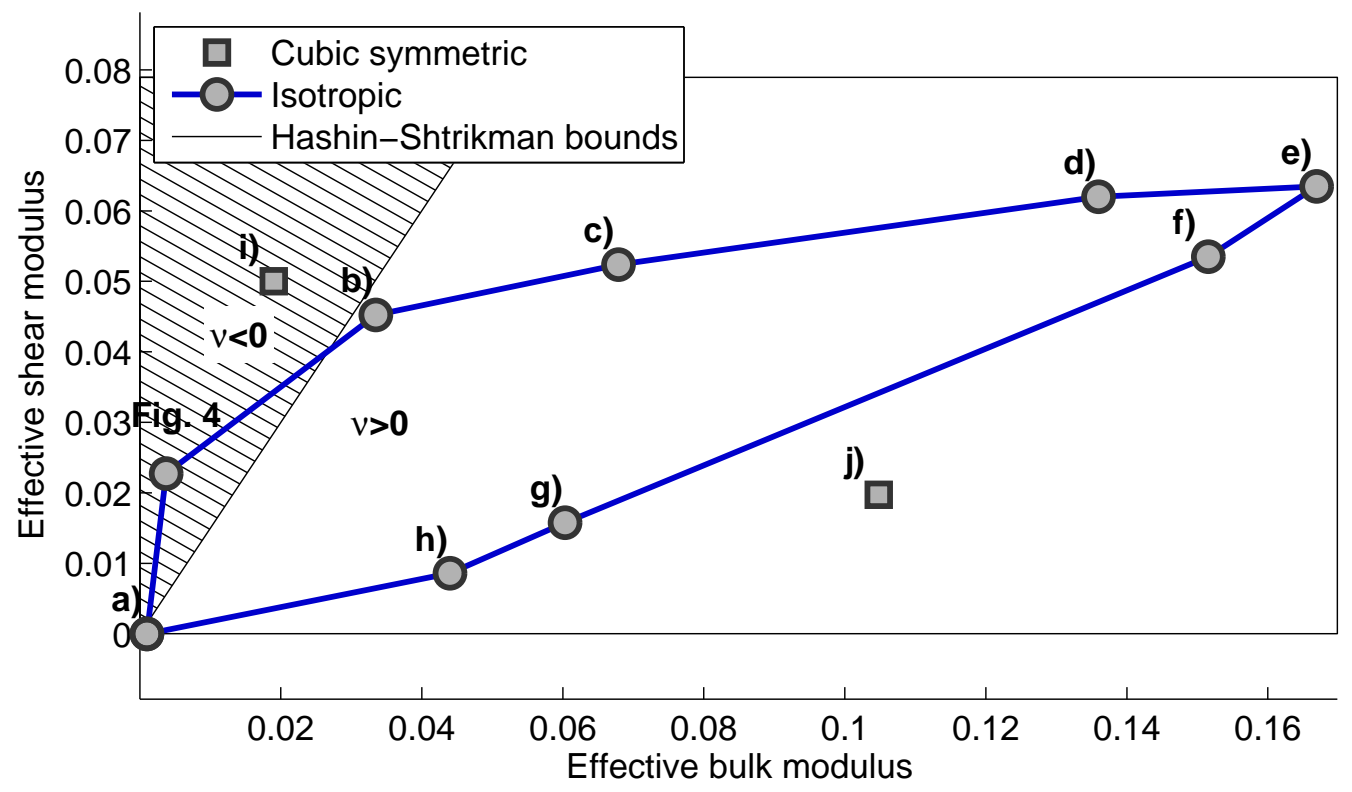

Figure 9: Hashin-Shtrikman bounds and effective moduli for several topology optimized designs. The base material properties are the same as for the structure in Figure 10, and the volume fraction of the blueprint design is constrained to $33.8 \%$ (corresponding to the design in Figure 10). Unit cells corresponding to the points are shown in Figure 10 .

stant number of iterations with increasing number of dofs. To save time in $3 \mathrm{D}$ we split the optimization into two parts. First, an optimization with a coarser mesh $\left(50^{3}\right.$ elements $)$ is performed. The result of this optimization is used as an initial guess for the fine mesh optimization. For the design presented in Figure 4 the number of coarse mesh iterations was around 3000, while the fine mesh optimization, with the coarse mesh initial guess, needed less than 500 iterations.

\section{Conclusion}

Topology optimization is now mature enough for optimization of directly manufacturable three-dimensional materials. With the presented method the optimized designs can directly be manufactured without any need for post-processing. This is especially important when working with threedimensional structures, since post-processing is hardly possible without de- 


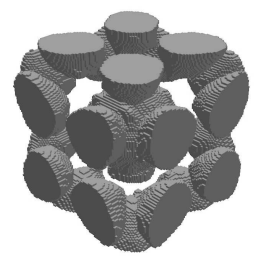

(a)

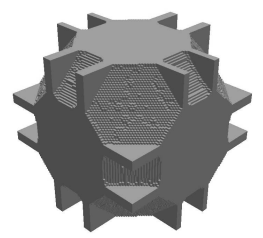

(f)

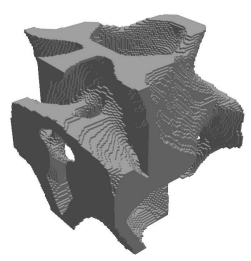

(b)

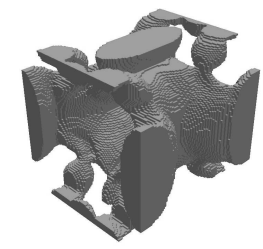

(g)

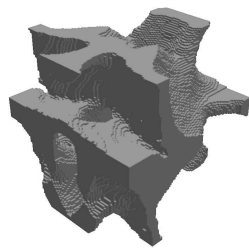

(c)

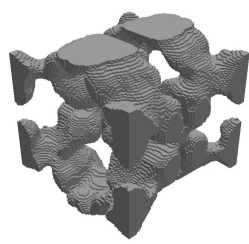

(h)

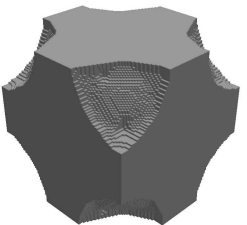

(d)

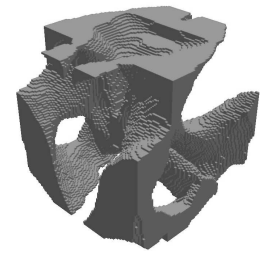

(i)

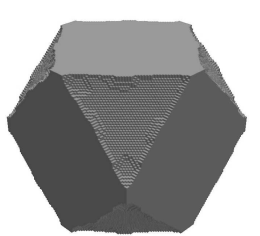

(e)

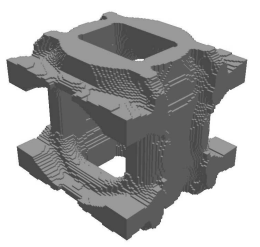

(j)

Figure 10: Unit cells for the extremal microstructures in Figure 9.

creasing performance or violating imposed constraints.

It is relatively easy to optimize for a stiff material, and with the presented method a one length-scale Vigdergauz like structure is obtained. The presented formulation can be used to optimize for other extremal elastic microstructures, such as negative Poisson's ratio, without any amendments. This is illustrated with the manufacturing of an isotropic material with a Poisson's ratio of -0.5 . Finally, it should be pointed out that the extension to multiphase materials is straightforward, however the resulting structures would require more complex manufacturing processes.

\section{Acknowledgement}

The work was funded by the Danish Research Agency through the innovation consortium F•MAT and the Villum Foundation through the NextTop project. The authors are grateful to the Product Development center at the Danish Technological Institute for manufacturing of the negative Poisson's ratio structure.

\section{Appendix A. Numerical homogenization}

Assuming a perfectly periodic material with infinite extent in all dimensions, the effective elasticity tensor can be computed by homogenization (e.g. 
Guedes and Kikuchi, 1990). Here, a description of the numerical procedure is presented in a compact form together with the gradients of the effective tensor components with respect to a design variable.

The domain occupied by a single periodic unit cell is denoted with $\Upsilon$. It is discretized using finite elements and the stiffness matrix of the discretized structure (Zienkiewicz et al., 2005) can be written as

$$
\mathbf{K}=\sum_{e=1}^{N} \int_{V_{e}} \mathbf{B}^{T} \mathbf{C}_{e} \mathbf{B} d V_{e}
$$

where $N$ denotes the finite elements number. The matrix $\mathbf{B}$ is the straindisplacement matrix, $\mathbf{C}_{e}$ is the constitutive matrix for the element, which in topology optimization will depends on the density of the element, and $V_{e}$ is the domain of element $e$.

To find the effective properties of the material the following finite element problem is solved with six load cases (three in 2D):

$$
\mathbf{K} \chi^{i}=\mathbf{f}^{i}, \quad i=1, \ldots, 6,
$$

where the displacement vectors $\chi^{i}$ are assumed to be $\Upsilon$ periodic, and the loads $\mathbf{f}^{i}$ correspond to unit strain fields computed as

$$
\mathbf{f}^{i}=\sum_{e} \int_{V_{e}} \mathbf{B}^{T} \mathbf{C}_{e} \varepsilon^{i}
$$

where the unit strains are

$$
\varepsilon^{1}=(1,0,0,0,0,0)^{T}, \quad \varepsilon^{2}=(0,1,0,0,0,0)^{T}, \quad \text { and so on }
$$

With the computed displacements, the homogenized constitutive matrix $\mathbf{C}^{*}$ can be found as

$$
\mathbf{C}^{*}=\frac{1}{|\Upsilon|} \sum_{e=1}^{N} \int_{V_{e}}\left(\mathbf{I}-\mathbf{B} \boldsymbol{\chi}_{e}\right)^{T} \mathbf{C}_{e}\left(\mathbf{I}-\mathbf{B} \boldsymbol{\chi}_{e}\right) d V_{e},
$$

where $\chi_{e}$ contains six columns corresponding to the six displacement fields, and $\mathbf{I}$ is a six times six identity matrix. The term $\mathbf{B} \boldsymbol{\chi}_{e}$ can be interpreted as the strains caused by the non-homogeneous material distribution.

Using the adjoint method it can be shown that the sensitivity of $\mathbf{C}^{*}$ with respect to change in an element design variable is: 


$$
\frac{\partial \mathbf{C}^{*}}{\partial \rho_{e}}=\frac{1}{|\Upsilon|} \sum_{e=1}^{N} \int_{V_{e}}\left(\mathbf{I}-\mathbf{B} \boldsymbol{\chi}_{e}\right)^{T} \frac{\partial \mathbf{C}_{e}}{\partial \rho_{e}}\left(\mathbf{I}-\mathbf{B} \boldsymbol{\chi}_{e}\right) d V_{e}
$$

\section{References}

Aage, N., Lazarov, B.S., 2013. Parallel framework for topology optimization using the method of moving asymptotes. Structural and Multidisciplinary Optimization 47, 493-505.

Andreassen, E., Clausen, A., Schevenels, M., Lazarov, B.S., Sigmund, O., 2011. Efficient topology optimization in matlab using 88 lines of code. Structural and Multidisciplinary Optimization 43, 1-16.

Bendsøe, M., Sigmund, O., 2003. Topology Optimization. Theory, Methods and Applications. Springer.

Berryman, J.G., Milton, G.W., 1988. Microgeometry of random composites and porous media. Journal of Physics D: Applied Physics 21, 87-94.

Cadman, J., Zhou, S., Chen, Y., Li, Q., 2012. On design of multi-functional microstructural materials. Journal of Materials Science 48, 1-16.

Cherkaev, A., Gibiansky, L., 1993. Coupled estimates for the bulk and shear moduli of a two-dimensional isotropic elastic composite. Journal of the Mechanics and Physics of Solids 41, 937-80.

Coelho, P.G., Cardoso, J.B., Fernandes, P.R., Rodrigues, H.C., 2011. Parallel computing techniques applied to the simultaneous design of structure and material. Advances in Engineering Software 42, 219-27.

Diaz, A., Sigmund, O., 2010. A topology optimization method for design of negative permeability metamaterials. Structural and Multidisciplinary Optimization 41, 163-77.

Gibiansky, L.V., Sigmund, O., 2000. Multiphase composites with extremal bulk modulus. Journal of the Mechanics and Physics of Solids 48, 461-98.

Guedes, J., Kikuchi, N., 1990. Preprocessing and postprocessing for materials based on the homogenization method with adaptive finite element methods. Computer Methods in Applied Mechanics and Engineering 83, 143-98. 
Guest, J.K., Prévost, J.H., 2006. Optimizing multifunctional materials: Design of microstructures for maximized stiffness and fluid permeability. International Journal of Solids and Structures 43, 7028-47.

Hashin, Z., Shtrikman, S., 1963. A variational approach to the theory of the elastic behaviour of multiphase materials. J. Mech. Phys. Solids (UK) 11, 127-40.

Jensen, J., Sigmund, O., 2011. Topology optimization for nano-photonics. Laser \& Photonics Reviews 5, 308-21.

Kadic, M., Bückmann, T., Stenger, N., Thiel, M., Wegener, M., 2012. On the practicability of pentamode mechanical metamaterials. Appl. Phys. Lett. (USA) 100, .

Kalpakjian, S., Schmid, S.R., Musa, H., 2010. Manufacturing engineering and technology. Pearson, Jurong, Singapore.

Krog, L., Tucker, A., Kemp, M., Boyd, R., 2004. Topology optimization of aircraft wing box ribs, in: Proceedings of The Altair Technology Conference.

Lakes, R., 1987. Foam structures with a negative Poisson's ratio. Science Science 235, 1038-40.

Lazarov, B.S., Schevenels, M., Sigmund, O., 2011. Robust design of largedisplacement compliant mechanisms. Mechanical Sciences 2, 175-82.

Milton, G.W., 1992. Composite materials with poisson's ratios close to -1 . Journal of the Mechanics and Physics of Solids 40, 1105-37.

Milton, G.W., Cherkaev, A., 1995. Which elasticity tensors are realizable? Trans. ASME, J. Eng. Mater. Technol. (USA) Journal of engineering materials and technology 117, 483-93.

Schwerdtfeger, J., Wein, F., Leugering, G., Singer, R.F., Körner, C., Stingl, M., Schury, F., 2011. Design of auxetic structures via mathematical optimization. Advanced Materials 23, 2650-4.

Sigmund, O., 1994. Materials with prescribed constitutive parameters: An inverse homogenization problem. International Journal of Solids and Structures 31, 2313-29. 
Sigmund, O., 2000a. A new class of extremal composites. Journal of the Mechanics and Physics of Solids 48, 397-428.

Sigmund, O., 2000b. Topology optimization: A tool for the tailoring of structures and materials. Philosophical Transactions of the Royal Society A: Mathematical, Physical and Engineering Sciences 358, 211-88.

Sigmund, O., 2007. Morphology-based black and white filters for topology optimization. Structural and Multidisciplinary Optimization 33, 401-24.

Sigmund, O., 2009. Manufacturing tolerant topology optimization. Acta Mechanica Sinica 25, 227-39.

Sigmund, O., Torquato, S., 1999. Design of smart composite materials using topology optimization. Smart Materials and Structures 8, 365-79.

Sigmund, O., Torquato, S., Aksay, I., 1998. On the design of 1-3 piezocomposites using topology optimization. Journal of Materials Research 13, 1038-48.

Svanberg, K., 1987. Method of moving asymptotes - a new method for structural optimization. International Journal for Numerical Methods in Engineering 24, 359-73.

Swan, C.C., Kosaka, I., 1997. Homogenization-based analysis and design of composites. Computers \& Structures 64, 603-21.

Theocaris, P.S., Stavroulakis, G.E., 1998. Multilevel optimal design of composite structures including materials with negative poisson's ratio. Structural Optimization 15, 8-15.

Vigdergauz, S.B., 1989. Regular structures with extremal elastic properties. Mechanics of Solids 24, 57-63.

Wang, F., Lazarov, B.S., Sigmund, O., 2011. On projection methods, convergence and robust formulations in topology optimization. Structural and Multidisciplinary Optimization 43, 767-84.

Zienkiewicz, O.C., Taylor, R.L., Zhu, J., 2005. The Finite Element Method. Elsevier. 